\title{
Estimação de Canais PLC Variante no Tempo na Presença de Ruído Impulsivo Aplicado a Sistemas OFDM
}

\author{
Antônio Ângelo M. Picorone, Augusto S. Cerqueira e Moisés V. Ribeiro
}

\begin{abstract}
Resumo-A presente contribuição tem como objetivo a investigação de técnicas de estimação de canais baseadas em sinais pilotos para modulação OFDM, quando o sistema multiportadora é aplicado para a transmissão de dados através de canais PLC (Power Line Communications). As técnicas analisadas foram os algoritmos Least square (LS) e Variable forgetting factor - Recursive Least square (RLS-VFF), interpoladores (nearest neighbour, spline, linear e cubic), equalizadores no domínio da frequência baseados nos critérios Zero forcing (ZF) e Minimum mean square error (MMSE). Resultados computacionais indicam que técnicas de estimação adaptativa combinadas com interpolador linear e equalizador baseado no critério MMSE são as que podem oferecer o melhor desempenho se a alocação dos sinais pilotos é retangular. Além disso, tais resultados evidenciam o fato de que o ruído AIGN (additive impulsive Gaussian noise) é o grande responsável pela redução considerável da capacidade de estimação de tais técnicas.
\end{abstract}

Palavras-Chave-power line communication, orthogonal frequency division multiplexing, estimação de canais, ruído impulsivo, canal variante no tempo.

Abstract-This paper aims at the investigation of pilot-aided channel estimation technique for OFDM modulation applied to PLC system. The analyzed techniques are Least square (LS) e Variable forgetting factor - Recursive Least square (RLS-VFF), interpolators based on nearest neighbor, spline, linear and cubic algorithms, frequency domain equalizers (FDE) based on Zero forcing (ZF) and Minimum mean square error (MMSE) criteria. Simulation results indicate that adaptive estimation technique combined with linear interpolation and FEQ based on MMSE criterion can offer the best performance if the rectangular grid for pilot allocation is considered. Moreover, these results reveal that the AIGN additive impulsive Gaussian noise is the main responsible for the performance degradation of the estimation technique.

Keywords-power line communication, orthogonal frequency division multiplexing, channel estimation, impulsive noise, timevarying channel.

\section{INTRODUÇÃO}

A modulação multiportadora baseada na OFDM (Orthogonal Frequency Division Multiplexing) juntamente com algoritmos bitloading têm sido aplicados em sistemas PLC (Powerline Communications), em que o canal de comunicação é o cabo de distribuição de energia elétrica, para maximizar o throughput dos mesmos [1]. Para garantir o sucesso de tal abordagem, a estimação da resposta em frequência dos canais

Antônio Ângelo M. Picorone, Augusto S. Cerqueira e Moisés V. Ribeiro são do Departamento de Energia Elétrica da Universidade Federal de Juiz de Fora, Minas Gerais, Brazil, E-mails: picorone@ieee.org, augusto.cerqueira@ufjf.edu.br, mribeiro@engenharia.ufjf.br
PLC se faz necessária. Em [2], [3] é analisado o problema de estimação de canais PLC de forma cega e supervisionada, a partir de sinais pilotos. Outros trabalhos [4], [5], [6], [7] analisam o mesmo problema de estimação de canais, porém em canais de comunicação sem fio.

No que tange sistemas PLC, entretanto, existem algumas questões de investigação ainda em aberto na literatura, tais como, $i$ ) a análise de desempenho em relação as estratégias de estimação do canal na posição dos pilotos, $i i)$ a geometria do piloto e sua alocação dinâmica e iii) as técnicas para obtenção da resposta em frequência do canal PLC a partir dos sinais pilotos. Tais questões de investigação assumem uma complexidade de análise maior se o canal PLC é linear $e$ variante no tempo (LVT) e corrompido pela presença de ruído impulsivo.

Objetivando apontar direções para possíveis respostas as questões de investigação supracitadas, nesta contribuição técnicas adaptativas e não adaptativas de estimação de canais, baseadas em sinais piloto, para modulação OFDM voltados para a transmissão de dados através de canais PLC LVT corrompidos pela presença de ruídos impulsivos aditivos são analisadas. Basicamente, os algoritmos LS e VFF-RLS (variable forgetting factor - recursive least square) são aplicados para estimar a resposta em frequência nas posições dos pilotos e, a seguir, diferentes algoritmos de interpolação (linear, cubic, spline e nearest) são aplicados. A análise de desempenho é fornecida a partir da BER (bit error rate) quando equalizadores baseados nos critérios ZF (zero forcing) and MMSE (minimum mean squared error) são aplicados. Diferentemente do que apontavam contribuições anteriores [2], os resultados computacionais sugerem um melhor desempenho do sistema OFDM quando a estimação do canal PLC LVT é baseada no algoritmo VFF-RLS, o interpolador é o linear e o equalizador é o MMSE. Além disso, tais resultados apontam que os ruídos impulsivos são os principais responsáveis pela perda de desempenho do sistema.

Este artigo está organizado da seguinte forma: na seção II, é formulado o problema da estimação de canais em ambientes PLC, na seção III, é descrito o sistema e o ambiente analisado, os resultados computacionais serão apresentados na seção IV e finalmente, na seção $\mathrm{V}$, serão apresentadas as conclusões deste trabalho.

\section{FORMULAÇÃO DO PROBLEMA}

Assumindo-se que $L_{p c} \geq L_{h}-1$, em que $L_{h}$ é o comprimento efetivo da versão discretizada de um modelo de canal de 
comunicação LVT e invariante durante um período de símbolo e $L_{p c}$ é o comprimento do prefixo cíclico (PC), então para um esquema de modulação OFDM, o vetor de dados na saída do canal, correspondente à transmissão de um símbolo OFDM, é dado por

$$
\mathbf{Y}(n)=\mathbf{C}(n) \mathbf{X}(n)+\mathbf{V}(n)
$$

em que $\mathbf{C}(n)=\operatorname{diag}\left\{H_{n}(0), H_{n}(1), \ldots, H_{n}(N-1)\right\}, \mathbf{V}(n)$ corresponde à DFT (discrete Fourier transform) do ruído aditivo na saída do canal de comunicação e $\mathbf{Y}(n)$ é o $n$ ésimo símbolo recebido. $H_{n}(i), i=0,1 \ldots, N-1$ são os coeficientes da DFT da resposta ao impulso do canal. $\mathbf{X}(n)$ e $\mathbf{Y}(n)$ são vetores colunas de comprimento $N$ e $\mathbf{X}(n)$ corresponde aos coeficientes da DFT do símbolo OFDM transmitido.

A estimativa do símbolo transmitido, a partir do processo de equalização, é dada por

$$
\begin{aligned}
\hat{\mathbf{X}}(n) & =\mathcal{W}(n) \mathbf{Y}(n) \\
& =\mathcal{W}(n) \mathbf{C}(n) \mathbf{X}(n)+\mathcal{W}(n) \mathbf{V}(n)
\end{aligned}
$$

em que $\mathcal{W}(n)=\operatorname{diag}\left\{W_{n}(0) W_{n}(1), \ldots, W_{n}(N-1)\right\}$ é o equalizador do sistema OFDM projetado assumindo-se que a estimativa do canal é baseada em sinais pilotos. De uma forma geral, o projeto do equalizador para um esquema de modulação OFDM, cuja estimação dos canais é baseada em sinais pilotos, pode ser dado por

$$
\mathcal{W}_{o}(n)=\min _{\hat{\mathbf{C}}(n, \Psi)} P_{b}
$$

em que $P_{b}$ é a probabilidade de erro de bit no receptor OFDM, $\Psi$ é o vetor de parâmetros que influenciam o projeto de $\hat{\mathbf{C}}(n)$, sendo $\hat{\mathbf{C}}(n)$ a estimativa do $\mathbf{C}(n)$. A formulação do problema de estimação de canais PLC baseando-se no critério de (3) evidencia que problema de estimação deve ser resolvido levando-se em conta que a estimativa do canal PLC deve contribuir para minimizar a $P_{b}$ no receptor. Assumindo-se que a estratégia para estimar a resposta em frequência do canal é função da inserção de pilotos nos símbolos transmitidos, então o equalizador ótimo pode ser obtido a partir de

$$
\mathcal{W}_{o}(n)=\min _{\hat{\mathbf{C}}(n, \alpha, \beta, \gamma, \theta)} P_{b}
$$

em que $\alpha=\left[\alpha_{1}, \ldots, \alpha_{N_{\alpha}}\right]$ refere-se às geometrias dos pilotos, $\beta=\left[\beta_{1}, \ldots, \beta_{N_{\beta}}\right]$ refere-se às técnicas de interpolações, $\gamma=$ $\left[\gamma_{1}, \ldots, \gamma_{N_{\gamma}}\right]$ refere-se às técnicas aplicadas para estimar os coeficientes de $\mathbf{C}(n)$ na posição do piloto $\hat{\mathbf{C}}_{p}(n)$ e $\theta$ referese ao critério aplicado ao equalizador. A minimização de (4) é um problema de otimização multiobjetivo de difícil solução. Assim sendo, a partir de escolhas em $\alpha, \beta, \gamma$ e que o critério para projeto do equalizador é o MMSE ou ZF, então simulações computacionais podem ser conduzidas para indicar soluções sub-ótimas que venham a fornecer curvas de desempenho satisfatórias para a análise da eficiência da modulação OFDM em sistemas PLC.

Considerando que o meio de comunicação é o cabo de distribuição de energia elétrica compreendido entre o secundário do transformador de baixa tensão e o medidor na entrada da casa do consumidor residencial, comercial ou industrial de energia elétrica, deve-se levar em conta que a resposta em frequência do mesmo pode ser expressa por [8]

$$
H(f, n)=\sum_{i=1}^{N_{r}} g_{i, n} e^{-\left(a_{0}+a_{1} f^{r}\right) d_{i}} e^{-j 2 \pi f \tau_{i}}
$$

em que $N_{r}$ é o número de percursos, $i$ refere-se ao $i$-ésimo percurso (o percurso com menor atraso recebe o índice $i=1$ ), $a_{0}$ e $a_{1}$ são os parâmetros de atenuação, $r$ é o expoente do fator de atenuação, $g_{i, n}$ é o fator de peso para o $i$-ésimo percurso para o $n$-ésimo símbolo. $d_{i}$ e $\tau_{i}$ é distância e o atraso do $i$-ésimo percurso, respectivamente.

A Figura 1 ilustra várias realizações do canal PLC quando $a_{0}=0, a_{1}=7,8.10^{-10}, r=1, \tau_{i}=d_{i} / 1,5.10^{8} \mathrm{e}$ $g_{i, n} \sim \mathcal{N}\left(\mu_{i}, .0001\right)$ em que $\mu_{i}$ são os valores indicados na Tabela I. Para os canais PLC, um modelo simplificado do

TABELA I

PARÂMETROS DO MODELO DO CANAL

\begin{tabular}{|c|c|c|c|c|}
\hline$i$ & 1 & 2 & 3 & 4 \\
\hline$d_{i}$ & 200.00 & 222.40 & 244.80 & 267.50 \\
$\mu_{i}$ & 0.64 & 0.38 & -0.15 & 0.05 \\
\hline
\end{tabular}

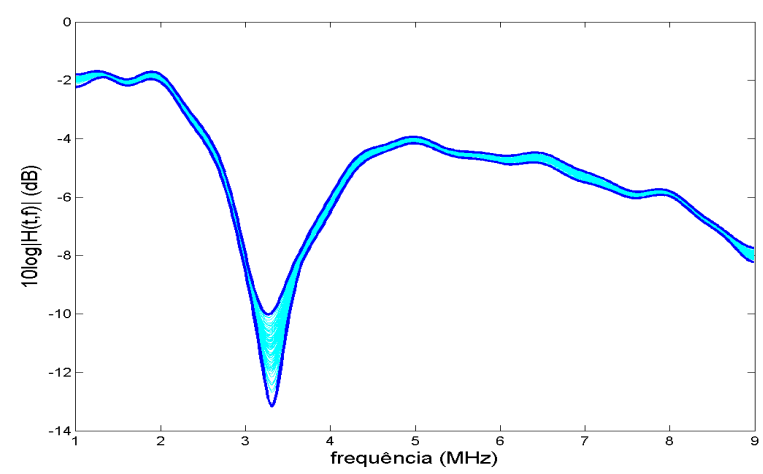

Fig. 1. Realizações do canal PLC LVT.

ruído aditivo e impulsivo na saída do canal PLC é dado por [9]

$$
v(n)=v_{\text {fundo }}(n)+v_{p s}(n)+v_{i m p}(n),
$$

em que $v_{\text {fundo }}(n) \sim \mathcal{N}\left(0, \sigma_{v}^{2}\right)$ representa o ruído de fundo, $v_{p s}(n) \sim \mathcal{N}\left(0,100 \sigma_{v}^{2}\right)$ representa o ruído impulsivo, periódico e síncrono em relação à frequência da rede elétrica. Finalmente, $v_{\text {imp }}(n) \sim \mathcal{N}\left(0,100 \sigma_{v}^{2}\right)$ representa o ruído impulsivo assíncrono. A duração de cada rajada de ruído impulsivo foi modelada como $t_{w, s}=100 \mu \mathrm{s}$. O tempo entre a ocorrência dos impulsos $t_{a r r, s}$ foi considerado como uma variável aleatória exponencial com média igual a $100 \mathrm{~ms}$ e $t_{w, s}=100 \mu \mathrm{s}$. Uma realização do ruído constituída pelos ruídos de fundo, impulsivo periódico e não periódico pode ser visto na Figura 2.

Para sistemas PLC, a questão de investigação ainda em aberto é a busca de $\hat{\mathbf{C}}(n)$ visando a obtenção de $\mathcal{W}(n)$ quando o canal é LVT e o ruído é aditivo e impulsivo. 


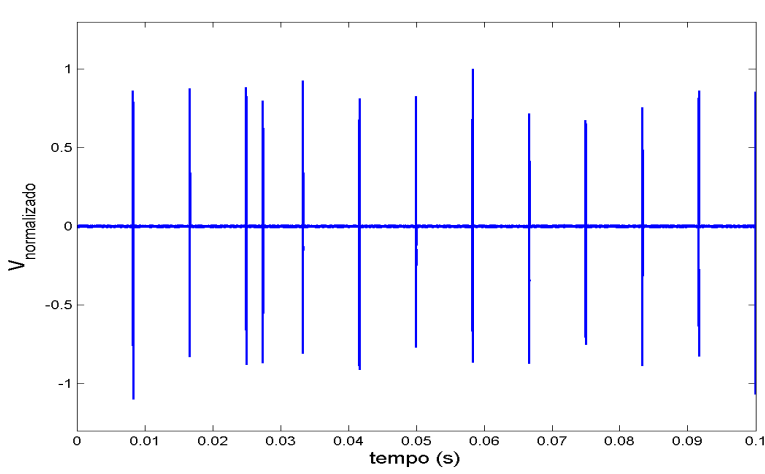

Fig. 2. Ruído impulsivo na saída do canal PLC LVT.

\section{Modelagem do Sistema}

Uma das formas de se estimar canais de comunicação é através da técnica que faz uso de pilotos, Data Aided [10]. Através dessa técnica, um símbolo OFDM completo ou parte dele, conhecido pelo receptor, é transmitido de tal forma que o receptor possa facilmente estimar o meio de comunicação através das amostras recebidas. A precisão da estimativa pode ser incrementada através do aumento da densidade dos pilotos. Entretanto, isto reduz a eficiência espectral [7]. A Figura 3 mostra o diagrama em blocos do receptor OFDM considerado para analisar as diferentes combinações de esquemas de estimação da resposta em frequência de canais PLC. O esquema apresentado baseia-se na inserção de pilotos no símbolo OFDM. Conforme é observado, o vetor de dados na saída da FFT é aplicado ao bloco de extração dos pilotos. Nas posições dos pilotos é estimado $\hat{\mathbf{C}}_{p}(n) \subset \hat{\mathbf{C}}(n)$, em que $\hat{\mathbf{C}}_{p}(n)=\operatorname{diag}\left\{\hat{C}_{p, n}(0) \hat{C}_{p, n}(1) \ldots \hat{C}_{p, n}(N-1)\right\}$. A partir de $\hat{\mathbf{C}}_{p}(n)$ e o emprego de técnicas de interpolação, obtém-se $\hat{\mathbf{C}}(n)$. Finalmente, o equalizador $\mathcal{W}(n)$ é obtido a partir de $\hat{\mathbf{C}}(n)$.

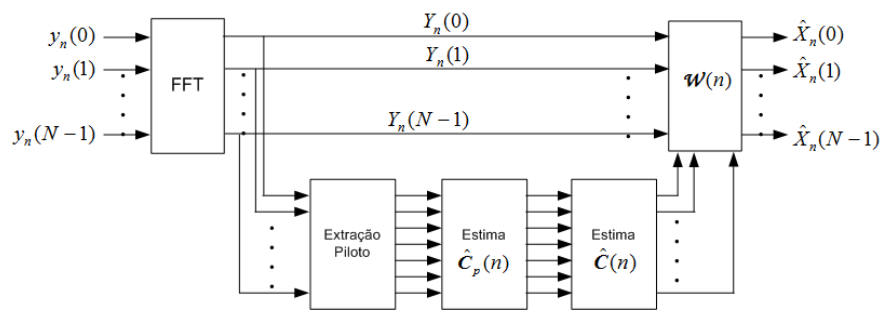

Fig. 3. Diagrama em blocos do sistema de estimação de canais PLC LVT na presença de ruído aditivo e impulsivo.

\section{A. Pilotos e sua Geometria}

A geometria retangular para inserção de sinais piloto nos símbolos OFDM é mostrada na Figura 4 para as 50 primeiras subportadoras. Neste caso, o espaçamento no tempo e frequência discretizada é igual a $D_{t}=1$ e $D_{f}=2$ [11]. Além disso, as portadoras 1 e $N$ sempre recebem sinais piloto.

\section{B. Estimação de $\hat{\mathbf{C}}_{p}(n)$}

Na técnica denominada Data-Aided, os $N_{p}$ sinais piloto $X_{p}(m), m=0,1, \ldots, N_{p}-1$ são uniformemente inseridos em

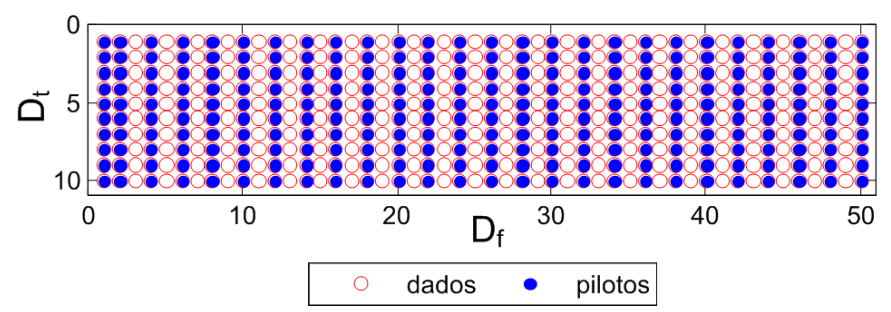

Fig. 4. Grid frequência $\times$ tempo de pilotos nos símbolos OFDM para $N=512, D_{t}=1$ e $D_{f}=2$.

$\mathbf{X}(n)$. Dessa forma, as $N$ subportadoras recebem os $N_{p}$ sinais piloto espaçados de $L=N / N_{p}$ subportadoras. A $k$-ésima subportadora do $n$-ésimo símbolo OFDM pode ser expressa como

$$
\begin{aligned}
X_{n}(k) & =X_{n}(m L+l) \\
& = \begin{cases}X_{p}(m), & l=0, \\
\text { dados de informação } & l=1,2, \ldots, L-1\end{cases}
\end{aligned}
$$

Considerando a resposta em frequência do canal PLC nas subportadoras que carregam os sinais piloto dada por

$$
\begin{aligned}
\mathbf{C}_{p}(n) & =\left[\begin{array}{ll}
C_{p, n}(0) & C_{p, n}(1) \ldots C_{p, n}\left(N_{p}-1\right)
\end{array}\right]^{T} \\
& =\left[\begin{array}{lll}
C(0) & C(L-1) \ldots C\left(\left(N_{p}-1\right) L-1\right)
\end{array}\right]^{T}
\end{aligned}
$$

em que foi omitido o subíndice $n$ referente ao $n$-ésimo símbolo OFDM para uma maior clareza. Então, pode-se expressar o vetor recebido dos sinais pilotos como,

$$
\begin{aligned}
\mathbf{Y}_{p}(n) & =\left[\begin{array}{ll}
Y_{p, n}(0) & Y_{p, n}(1) \ldots Y_{p, n}\left(N_{p}-1\right)
\end{array}\right]^{T} \\
\mathbf{Y}_{p}(n) & =\mathbf{C}_{p}(n) \mathbf{X}_{p}(n)+\mathbf{V}_{p}(n)
\end{aligned}
$$

em que $\mathbf{V}_{p}(n)$ é um vetor representando o ruído em cada subportadora piloto. Para a obtenção de $\hat{\mathbf{C}}_{p}(n)$ a partir de $\mathbf{Y}_{p}(n)$, diversas técnicas de estimação podem ser aplicadas, dentre as quais destacam-se as baseadas na abordagem adaptativa e nãoadaptativas. Dentre as várias, no presente trabalho as técnicas LS e VFF-RLS foram escolhidas. A primeira, por ser uma técnica simples e tradicionalmente aplicada nestes casos [7], a segunda, por ser uma versão do algoritmo RLS cujo fator de esquecimento varia com a dinâmica do sistema e, consequentemente, apresenta maior velocidade de convergência. O estimador LS fornece

$$
\hat{C}_{p, n}(k)=\frac{Y_{n}(k)}{X_{n}(k)}=C_{n}(k)+\frac{V_{n}(k)}{X_{n}(k)},
$$

em que $k=0,1, \ldots, N_{p}-1$. A versão do algoritmo RLS quando o fator de esquecimento é variável é chamada de VFF- 
RLS, cujas equações são dadas por [5]

$$
\begin{aligned}
e_{n}(k)= & Y_{n}(k)-\hat{C}_{p, n-1}(k) X_{n}(k) \\
q_{n}(k)= & \frac{P_{n-1}(k) X_{n}(k)}{\lambda \sigma_{n, k}^{2}+Y_{n}^{*}(k) P_{n-1}(k) Y_{n}(k)} \\
\hat{C}_{p, n}(k)= & \hat{C}_{p, n-1}(k)+q_{n}(k) e_{n}^{*}(k) \\
P_{n}(k)= & \frac{1}{\lambda_{n}}\left[P_{n-1}(k)-q_{n}(k) X_{n}^{*}(k) P_{n-1}(k)\right] \\
M_{n}(k)= & \frac{1}{\lambda_{n}(k)}\left[1-q_{n}(k) X_{n}^{*}(k)\right] M_{n-1}(k) \times \\
& {\left[1-X_{n}(k) q_{n}^{*}(k)\right]+\left(\frac{1}{\lambda_{n}(k) \sigma_{n, k}^{2}}\right) \times } \\
& q_{n}(k) q_{n}^{*}(k)-\frac{1}{\lambda_{n}(k) P_{n}(k)} \\
D_{n}(k)= & {\left[1-q_{n}(k) X_{n}^{*}(k)\right] D_{n-1}(k)+} \\
& M_{n}(k) S_{n}(k) \frac{e_{n}^{*}(k)}{\sigma_{n, k}^{2}} \\
& {\left[\lambda_{n}(n-1)+\frac{\alpha}{\sigma_{n, k}^{2}} \times\right.} \\
\left.\lambda_{n}(k)\left\{D_{n-1}^{*}(k) X_{n}(k) e_{n}^{*}(k)\right\}\right]_{\lambda_{-}}^{\lambda_{+}} & \mathbb{R},
\end{aligned}
$$

em que $k$ é o $k$-ésimo coeficiente na diagonal principal de $\hat{\mathbf{C}}_{p}(n),(\cdot)^{*}$ representa o conjugado, o parâmetro $\alpha$ é a taxa de aprendizagem, o coeficiente $D_{n}(k)$ é o gradiente, o coeficiente $M_{n}(k)$ é a inversa do coeficiente de correlação $P_{n}(k)$. Nesse algoritmo, a atualização do fator de esquecimento $\left(\lambda_{n}\right)$ contém o efeito do ruído $\left(\sigma_{n, k}^{2}\right)$ em cada subportadora, que contribui para o rastreamento das variações do canal.

\section{Estimação de $\mathbf{C}(n)$}

A estimativa de $\mathbf{C}(n)$ é obtida interpolando-se os pontos entre os coeficientes de $\hat{\mathbf{C}}_{p}(n)$. Dentre várias técnicas de interpolação, as seguintes foram utilizadas no presente trabalho: linear, cúbica, spline e mais próxima.

No método de interpolação linear, duas sucessivas subportadoras piloto são utilizadas para determinar o coeficiente da resposta em frequência do canal nas subportadoras que estão localizadas entre os pilotos [4]. Para a subportadora de dados $k, m L \leq k<(m+1) L$, a resposta do canal estimado usando o método de interpolação linear é dado por

$$
\begin{aligned}
\hat{C}_{n}(k)= & \hat{C}_{n}(m L+l) \\
= & \left(1-\frac{l}{L}\right) \hat{C}_{p, n}(m)+\frac{l}{L} \hat{C}_{p, n}(m+1) \\
= & \hat{C}_{p, n}(m)+\frac{l}{L}\left(\hat{C}_{p, n}(m+1)-\hat{C}_{p, n}(m)\right) \\
& , 0 \leq l<L,
\end{aligned}
$$

A interpolação baseada em spline é dada por

$$
\begin{aligned}
\hat{C}_{n}(k)= & \hat{C}_{n}(m L+l) \\
= & C_{1} \hat{C}_{p, n}(m-1)+C_{0} \hat{C}_{p, n}(m)+ \\
& C_{-1} \hat{C}_{p, n}(m+1), \\
& \left\{\begin{array}{l}
C_{1}=\frac{\xi(\xi+1)}{2} \\
C_{0}=-(\xi-1)(\xi+1) \\
C_{-1}=\frac{\xi(\xi-1)}{2}
\end{array}\right.
\end{aligned}
$$

e $\xi=l / N$.

$\mathrm{Na}$ interpolação cúbica o coeficiente da função de transferência do canal PLC em cada subportadora é aproximado por um polinômio de terceira ordem com respeito a $l / L$. A estimativa nas subportadoras é [6]:

$$
\begin{aligned}
\hat{C}_{n}(k)= & A\left(\frac{l}{L}\right) \hat{C}_{p, n}(m)+B(l / L)\left(\frac{l}{L}\right)+\hat{C}_{p, n}(m+1)+ \\
& +C\left(\frac{l}{L}\right) z(m)+D\left(\frac{l}{L}\right) z(m+1)
\end{aligned}
$$

em que $A\left(\frac{l}{L}\right), B\left(\frac{l}{L}\right), C\left(\frac{l}{L}\right)$ e $D\left(\frac{l}{L}\right)$ são constantes determinadas por $l / L . z(m)=C_{p}^{\prime \prime}(m)$ é a derivada de segunda ordem da função de transferência do $m$-ésimo sinal de referência.

A interpolação pelo método mais próximo (nearest) é descrita como a técnica em que o coeficiente da resposta em frequência do canal é aproximado para o valor mais próximo dos sinais piloto. É o método mais rápido e simples, mas que usualmente fornece os piores resultados.

\section{Equalização de $\mathbf{Y}(n)$}

Baseando-se no critério ZF, então o equalizador é $\mathcal{W}(n)=$ $\hat{\mathbf{C}}^{-1}(n)$. Por outro lado, a equalização a partir do critério MMSE fornece [12]

$$
\mathcal{W}(n)=\left[\hat{\mathbf{C}}^{H}(n) \hat{\mathbf{C}}(n)+\boldsymbol{\Lambda}^{-1}(n)\right]^{-1} \hat{\mathbf{C}}^{H}(n)
$$

em que $\boldsymbol{\Lambda}(n)=\operatorname{diag}\left\{\frac{\sigma_{s, 0}^{2}}{\sigma_{n, 0}^{2}}, \frac{\sigma_{s, 1}^{2}}{\sigma_{n, 1}^{2}}, \ldots, \frac{\sigma_{s, N-1}^{2}}{\sigma_{n, N-1}^{2}}\right\},(\cdot)^{H}$ é o operador Hermitiano, $\sigma_{s, k}^{2}$ e $\sigma_{n, k}^{2}$ são as variâncias do sinal transmitido e ruído na $k$-ésima subportadora do receptor.

\section{RESULTADOS COMPUTACIONAIS}

Um sistema OFDM com $N$ subportadoras possui um comprimento igual a $2 N+L_{p c}$ amostras. Dessa forma, o tempo necessário para a transmissão de cada símbolo OFDM $\left(t_{s i m b}\right)$ pode ser expresso por:

$$
t_{\text {simb }}=\left(2 N+L_{p c}\right) / 2 B \quad(\text { segundos }),
$$

em que $B$ representa a largura de banda do canal de comunicação. Resultados obtidos em [13] revelam que o tempo de coerência $\left(t_{c o e r}\right)$ do canal PLC pode atingir valores próximos de $600 \mu \mathrm{s}$, o que nos leva a considerar o comportamento do canal como invariante no tempo durante a transmissão de $t_{\text {coer }} / t_{\text {simb }}$ símbolos OFDM.

Os parâmetros utilizados para o sistema PLC baseado na modulação OFDM estão indicados na Tabela II. Nas figuras de desempenho, $E_{b} / N_{0}$ refere-se a relação entre a energia do símbolo transmitido e a densidade espectral de potência do ruído de fundo, $v_{b k g r}(n)$. O canal PLC LVT tem a energia normalizada. Além disso, considerou-se o modelo do ruído aditivo como sendo puramente gaussiano (AWGN) e aquele descrito na seção II (AIGN- Additive Impulsive Gaussian Noise). Os parâmetros de inicialização e limites do fator de esquecimento são mostrados na Tabela III.

As figuras 5 e 6 sugerem um melhor desempenho do interpolador linear, seguido pelo spline, tanto no ambiente com ruído AWGN quanto com ruído impulsivo AIGN, quando se utiliza o equalizador baseado no critério ZF. Pode-se observar 
TABELA II

PARÂMETROS UTILIZADOS NA SIMULAÇÃo

\begin{tabular}{|c|c|}
\hline Descrição & Valor \\
\hline Quantidade de subportadoras & 512 \\
\hline Tamanho do prefixo cíclico & 32 \\
\hline frequência mínima & $1 \mathrm{MHz}$ \\
\hline frequência máxima & $9 \mathrm{MHz}$ \\
\hline Modulação & BPSK \\
\hline Tempo de coerência do canal & $600 \mu s$ \\
\hline Téc. de Estimação & LS e VFF-RLS \\
\hline Téc. de Interpolação & linear, spline, cúbica, mais próxima \\
\hline Téc. de Equalização & ZF e MMSE \\
\hline Geom. do grid & retangular \\
\hline
\end{tabular}

TABELA III

PARÂMETROS DO VFF-RLS

\begin{tabular}{|c|c|}
\hline Descrição & Valor \\
\hline Fator de esquecimento $\lambda_{i n i}$ & .6 \\
\hline$\lambda_{+}$ & .999 \\
\hline$\lambda_{-}$ & .4 \\
\hline Passo de adaptação $\alpha$ & .01 \\
\hline
\end{tabular}

também que o estimador VFF-RLS apresenta uma menor $E_{b} / N_{o}$ para mesma BER comparado com o estimador LS. Além disso, o estimador VFF-RLS parece não apresentar uma discrepância muito grande na BER quando se modifica o tipo de interpolador. A estabilização da BER quando $E_{b} / N_{o}$ é de aproximadamente 25 a $30 \mathrm{~dB}$ é devido ao ruído AIGN.

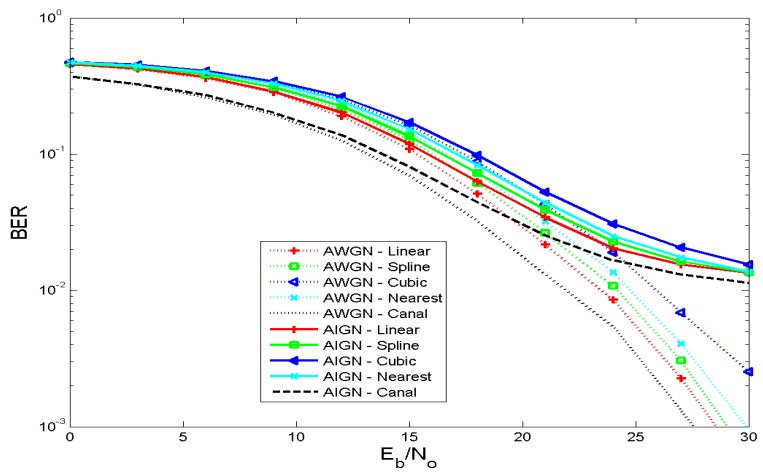

Fig. 5. BER obtida com o estimador LS e o equalizador ZF para AWGN e AIGN para diferentes tipos de interpoladores.

As Figuras 7 e 8 também sugerem que o melhor desempenho, do ponto de vista da interpolação, é obtido pelo interpolador linear, seguido pelo spline, considerando os ambientes com ruído AWGN e AIGN e utilizando-se o equalizador baseado no critério MMSE. Todas as observações feitas quando se utiliza o equalizador ZF são válidas para o MMSE, ou seja, no geral, os melhores desempenhos são alcançados quando o estimador é o VFF-RLS. Dado que a modulação é BPSK, os critérios MMSE e ZF fornecem o mesmo desempenho do ponto de vista de simulação, posto que ambos corrigem de forma apropriada a distorção de fase introduzida pelo canal PLC.

A figura 9 mostra uma comparação do desempenho entre os estimadores LS e VFF-RLS quando se utiliza o interpolador linear e o equalizador MMSE. Esse resultado aponta para um melhor desempenho do conjunto estimador VFF-RLS,

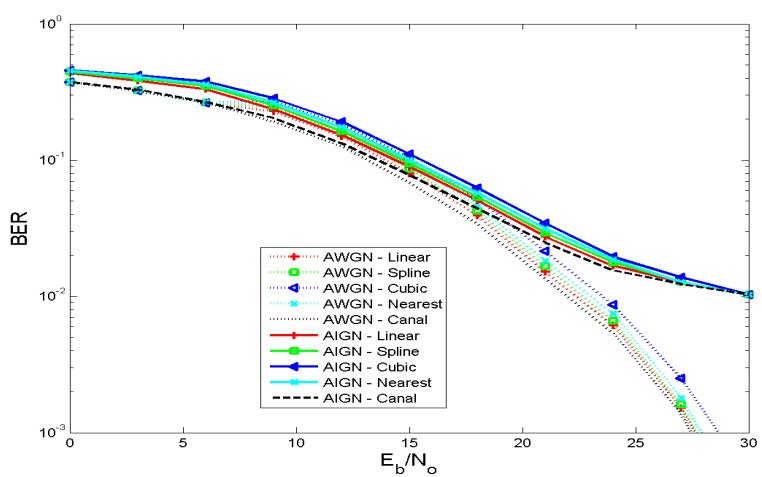

Fig. 6. BER obtida com o estimador VFF-RLS e o equalizador ZF para AWGN e AIGN para diferentes tipos de interpoladores.

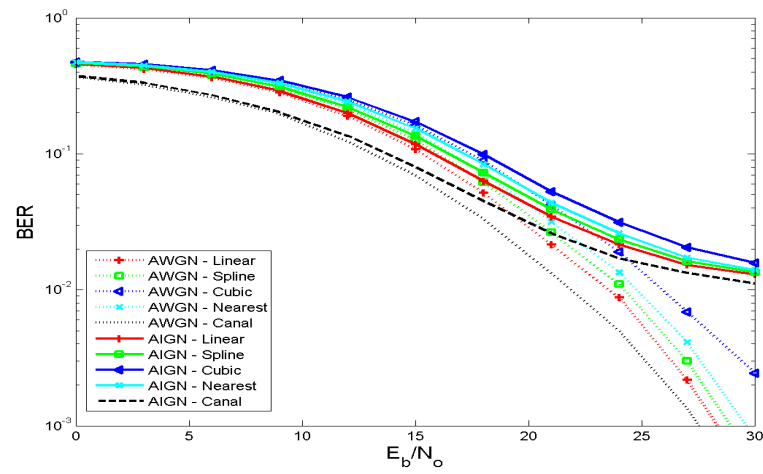

Fig. 7. BER obtida com o estimador LS e o equalizador MMSE para AWGN e AIGN para diferentes tipos de interpoladores.

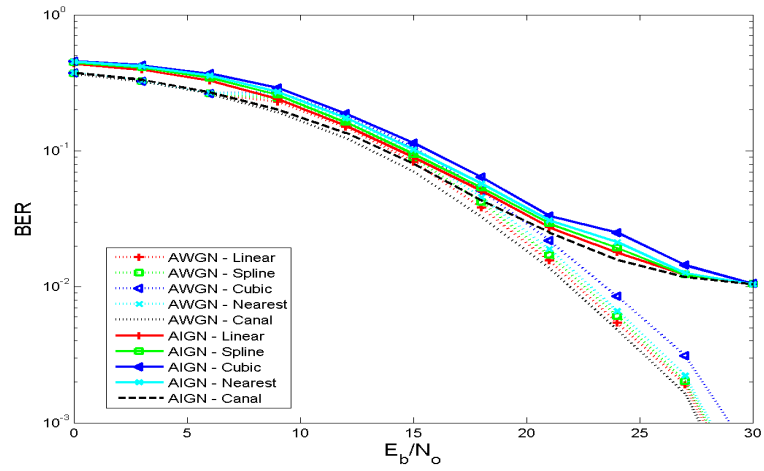

Fig. 8. BER obtida com o estimadores VFF-RLS e o equalizador MMSE para AWGN e AIGN para diferentes tipos de interpoladores.

interpolador linear e equalização MMSE. Pode-se observar que, para qualquer BER escolhida, encontra-se no estimador VFF-RLS em relação ao LS uma menor BER, para ambos os tipos de ruídos (AWGN e AIGN).

A evolução de um dos coeficientes da resposta em frequência do canal e do canal estimado, após a convergência do algoritmo adaptativo está representada na figura $10\left(E_{b} / N_{o}=30 \mathrm{~dB}\right)$. Observa-se nesta figura um melhor rastreamento na estimativa de $\mathbf{C}(n)$ quando se utiliza o estimador adaptativo, o que corrobora com os resultados obtidos em ambientes wireless. Os picos próximos aos símbolos 705 


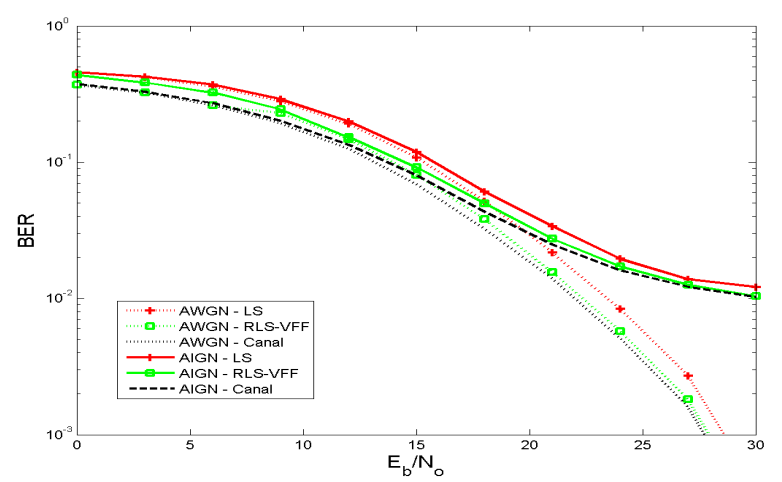

Fig. 9. BER obtida com os estimadores adaptativo e não-adaptativo, interpolador linear e equalizador MMSE.

e 770 são devidos ao ruído AIGN.

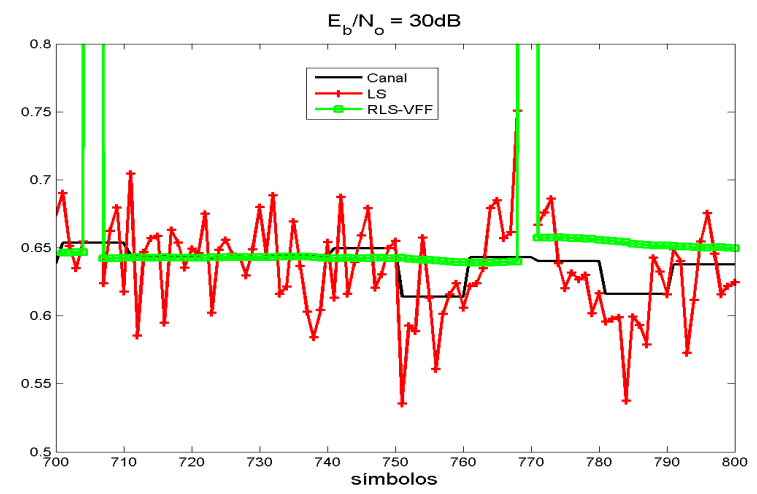

Fig. 10. Convergência dos estimadores LS e RLS-VFF associados a um coeficiente de $\mathbf{C}(n)\left(E_{b} / N_{o}=30\right)$.

\section{CONCLUSÕES}

Este trabalho investigou o uso das técnicas de estimação LS e VFF-RLS de canais baseadas em sinais piloto para modulações OFDM aplicada a canais PLC LVT e corrompidos pela presença de AIGN. Simulações computacionais indicam um melhor desempenho do sistema PLC quando a estimação de $\mathbf{C}(n)$ é baseada no algoritmo VFF-RLS, o interpolador é o linear e o equalizador é o MMSE. Tal resultado indica que estimadores adaptativos são capazes de fornecer melhores estimativas dos canais PLC LVT. A constatação de que o interpolador linear é o mais adequado vai de encontro ao que tem sido apresentado na literatura. Finalmente, os resultados computacionais apontam que a redução considerável do desempenho do sistema PLC baseado na modulação OFDM é devida à presença de ruídos impulsivos. Tal constatação indica que uma estratégia adequada para estimação confiável dos canais PLC LVT é predizer os intervalos de tempo em que os ruídos impulsivos ocorrem e utilizar técnicas que possam minimizar a influência dos mesmos.

\section{AGRADECIMENTOS}

Este trabalho foi apoiado pelo CNPq, CAPES, FAPEMIG, FINEP e INERGE.

\section{REFERÊNCIAS}

[1] M. V. Ribeiro, "Power line communications: a promising communication system's paradigm for last miles and last meters applications," in Telecommunications: Advances and Trends in Transmission, Networking and Applications, C. C. Cavalcante, R. F. Colares, and P. C. Barboza, Eds. Fortaleza: Univ. Fortaleza Press, 2006, pp. 133-1 56.

[2] J. Cortes, A. Tonello, and L. Diez, "Comparative analysis of pilotbased channel estimators for DMT systems over indoor power-line channels," in IEEE Inter. Symp. on Power Line Communcations and Its Applications, 2007, pp. 372-377.

[3] A. Musolino, M. Raugi, and M. Tucci, "Cyclic short-time varying channel estimation in OFDM power-line communication," IEEE Trans. Power Delivery, vol. 23, no. 1, pp. 157-163, Jan. 2008.

[4] M.-H. Hsieh and C.-H. Wei, "Channel estimation for OFDM systems based on comb-type pilot arrangement in frequency selective fading channels," IEEE Trans. Cons. Electr., vol. 44, no. 1, pp. 217-225, Feb. 1998.

[5] S. Song, J.-S. Lim, S. J. Baek, and K.-M. Sung, "Variable forgetting factor linear least squares algorithm for frequency selective fading channel estimation," IEEE Trans. Vehicular Techn., vol. 51, no. 3, pp. 613-616, May 2002.

[6] S. G. Kang, Y. M. Ha, and E. K. Joo, "A comparative investigation on channel estimation algorithms for OFDM in mobile communications," IEEE Trans. Broadc., vol. 49, no. 2, pp. 142-149, June 2003.

[7] H. Ozdemir, M.K.; Arslan, "Channel estimation for wireless OFDM systems," IEEE Commun. Surveys \& Tutorials, vol. 9, no. 2, pp. 18-48, 2nd Quarter 2007.

[8] M. Zimmermann and K. Dostert, "A multipath model for the powerline channel,” IEEE Trans. Commun., vol. 50, no. 4, pp. 553-559, Apr 2002.

[9] R. Hormis, I. Berenguer, and X. Wang, "A simple baseband transmission scheme for power line channels," IEEE J. Sel. Areas in Commun., vol. 24, no. 7, pp. 1351-1363, July 2006.

[10] S. Colieri, M. Ergen, A. Puri, and B. A, "A study of channel estimation in OFDM systems," in Proc. IEEE Vehicular Techn. Conf., vol. 2, 2002, pp. 894-898.

[11] M.-G. Garcia, S. Zazo, and J. Paez-Borrallo, "Pilot patterns for channel estimation in OFDM," Electronics Letters, vol. 36, no. 12, pp. 10491050, June 2000.

[12] A. Tajer and A. Nosratinia, "Diversity order of MMSE single-carrier frequency domain linear equalization," in IEEE Global Telecom. Conf., 2007, pp. 1524-1528.

[13] F. Corripio, J. Arrabal, L. del Rio, and J. Munoz, "Analysis of the cyclic short-term variation of indoor power line channels," IEEE J. Sel. Areas in Commun., vol. 24, no. 7, pp. 1327-1338, July 2006. 